\title{
Acutely altered mental status as the main clinical presentation of multiple strokes in critically ill patients with COVID-19
}

\author{
Carolina Díaz-Pérez ${ }^{1}$ (1) $\cdot$ Carmen Ramos $^{1}$ - Alberto López-Cruz ${ }^{2}$. José Muñoz Olmedo ${ }^{3}$. Jimena Lázaro González ${ }^{4}$. \\ Enrique De Vega-Ríos ${ }^{5}$. Carmen González-Ávila ${ }^{1} \cdot$ Carlos Hervás $^{1} \cdot$ Santiago Trillo ${ }^{1}$ • José Vivancos ${ }^{1}$
}

Received: 7 May 2020 / Accepted: 7 August 2020 / Published online: 17 August 2020

(C) Fondazione Società Italiana di Neurologia 2020

\begin{abstract}
Background and aims Cerebral infarction in COVID-19 patients might be associated with a hypercoagulable state related to a systemic inflammatory response. Its diagnosis might be challenging. We present two critically ill patients with COVID-19 who presented acutely altered mental status as the main manifestation of multiple strokes.

Methods Clinical presentation and diagnostic work-up of the patients.

Results Two patients in their sixties were hospitalized with a bilateral pneumonia COVID-19. They developed respiratory failure and were admitted to ICU for mechanical ventilation and intense medical treatment. They were started on low-molecular-weight heparin since admission. Their laboratory results showed lymphopenia and increased levels of C-reactive protein and D-dimer. Case 1 developed hypofibrinogenemia and presented several cutaneous lesions with biopsy features of thrombotic vasculopathy. Case 2 was performed a CT pulmonary angiogram at ICU showing a bilateral pulmonary embolism. When waking up, both patients were conscious but with a remarkable global altered mental status without focal neurological deficits. A brain MRI revealed multiple acute bilateral ischemic lesions with areas of hemorrhagic transformation in both patients (case 1: affecting the left frontal and temporal lobes and both occipital lobes; case 2: affecting both frontal and left occipital lobes). Cardioembolic source and acquired antiphospholipid syndrome were ruled out. COVID-19-associated coagulopathy was suspected as the possible main etiology of the strokes.

Conclusion Acutely altered mental status might be the main manifestation of multiple brain infarctions in critically ill COVID-19 patients. It should be specially considered in those with suspected COVID-19-associated coagulopathy. Full-dose anticoagulation and clinical-radiological monitoring might reduce their neurological consequences.
\end{abstract}

Keywords COVID-19 $\cdot$ Acutely altered mental status $\cdot$ Multiple strokes

Carolina Díaz-Pérez and Carmen Ramos contributed equally to this work.

Carolina Díaz-Pérez

carodipez@gmail.com

1 Department of Neurology, La Princesa University Hospital, Madrid, Spain

2 Department of Psychiatry, La Princesa University Hospital, Madrid, Spain

3 Neuroradiology Unit, Department of Radiology, La Princesa University Hospital, Madrid, Spain

4 Intensive Care Unit, La Princesa University Hospital, Madrid, Spain

5 Department of Internal Medicine, La Princesa University Hospital, Madrid, Spain

\section{Introduction}

The COVID-19 caused by the novel coronavirus SARS-CoV2 usually manifests with fever, cough, and dyspnea, with characteristic bilateral areas of ground-glass opacity on chest CT images. Some patients progress to an inflammatory phase that might require mechanical ventilation and admission to the intensive care unit (ICU) [1].

In severe forms of the disease, several neurological manifestations have been described, mostly affecting the central nervous system [2]. Cerebral infarction may be associated in these patients with a hypercoagulable state due to a systemic inflammatory response [3-5].

It is not unusual for critically ill patients to develop brain ischemic lesions with symptoms not clearly identified as a focal neurological deficit. We present two critically ill 
COVID-19 patients who presented acutely altered mental status as the main manifestation of multiple strokes.

\section{Results}

\section{Case 1}

A 61-year-old-man presented with a bilateral pneumonia COVID-19 with fever, cough, and dyspnea. He was admitted to the Internal Medicine Service on hydroxychloroquine, lopinavir/ritonavir, and low-molecular-weight heparin (LMWH) $60 \mathrm{mg}$ q.d.

He developed respiratory failure and the laboratory results showed lymphopenia (300 cells/ $\mu$ l [normal 10004000]) and increased levels of C-reactive protein (32.2 $\mathrm{mg} / \mathrm{dl}$ [normal < 0.50]), ferritin $(538 \mathrm{ng} / \mathrm{ml}$ [normal 30$400]$ ), fibrinogen (631 mg/dl [normal 150-400]), and Ddimer $(124.86 \mu \mathrm{g} / \mathrm{ml}$ [normal $0.15-0.50])$. He was admitted to the ICU for mechanical ventilation, receiving systemic corticosteroids and tocilizumab. A remarkable complication was hypofibrinogenemia (73 $\mathrm{mg} / \mathrm{dl})$ with prolonged prothrombin time (18.6 s [normal 0-13.9]) and slight thrombocytopenia $(99,000$ cells/ $\mu 1$ [normal 150,000-400,000]), consistent with disseminated intravascular coagulation according to the International Society on Thrombosis and Haemostasis criteria [6].

After clinical and analytical improvement, he was discharged back to the Internal Medicine Service. Neurological examination revealed drowsiness, inattention, disorientation, and slow speech with no focal neurologic deficits. The symptoms fluctuated and psychomotor agitation partially responded to quetiapine. The electroencephalogram pattern was suggestive of moderate diffuse encephalopathy.

A brain MRI was performed demonstrating multiple ischemic lesions affecting the left frontal and temporal lobes and both occipital lobes with extensive areas of hemorrhagic transformation (Fig. 1a-c). Cardioembolic source was ruled out and acquired antiphospholipid syndrome study was normal. He developed several cutaneous lesions with biopsy features of thrombotic vasculopathy. These findings, together with the analytic disturbances, pointed to COVID-19-associated coagulopathy as the main etiology suspected for the strokes.

\section{Case 2}

A 65-year-old man with hypertension and diabetes mellitus presented with a bilateral pneumonia COVID-19 with fever and dyspnea, mild respiratory compromise, and analytic disturbances (lymphopenia of $790 \mathrm{cells} / \mu \mathrm{l}$ [normal 1000-4000], D-dimer $0.55 \mu \mathrm{g} / \mathrm{ml}$ [normal $0.15-0.50$ ], C-reactive protein $10.2 \mathrm{mg} / \mathrm{dl}$ [normal < 0.50], lactate dehydrogenase $338 \mathrm{U} / 1$ [normal 135-225]). He was admitted to the Internal Medicine Service on hydroxychloroquine, lopinavir/ritonavir, and LMWH $80 \mathrm{mg}$ b.i.d.

He developed respiratory failure with analytical deterioration (lymphopenia of 410 cells $/ \mu \mathrm{l}$, D-dimer $21^{\prime} 4 \mu \mathrm{g} / \mathrm{ml}$, lactate dehydrogenase $820 \mathrm{U} / 1$ ). He was admitted to ICU for mechanical ventilation, receiving high doses of methylprednisolone and tocilizumab. Due to persistent high D-dimer levels, a CT pulmonary angiogram was performed showing a bilateral pulmonary embolism. He was discharged back to the Internal Medicine Service 2 weeks later, remaining respiratory stable with analytical improvement.
Fig. 1 a, b Case 1. Multiple ischemic lesions visible on DWI sequences (brain MRI) affecting the left frontal and temporal lobes and both occipital lobes. c Case 1 . Extensive areas of hemorrhagic transformation on SWI sequences (brain MRI) affecting both occipital lobes. d, e Case 2. Acute bilateral ischemic lesions affecting both frontal and left occipital lobes on DWI sequences. f Case 2. Several microhemorrhagic foci affecting both frontal and left occipital lobes on SWI sequences

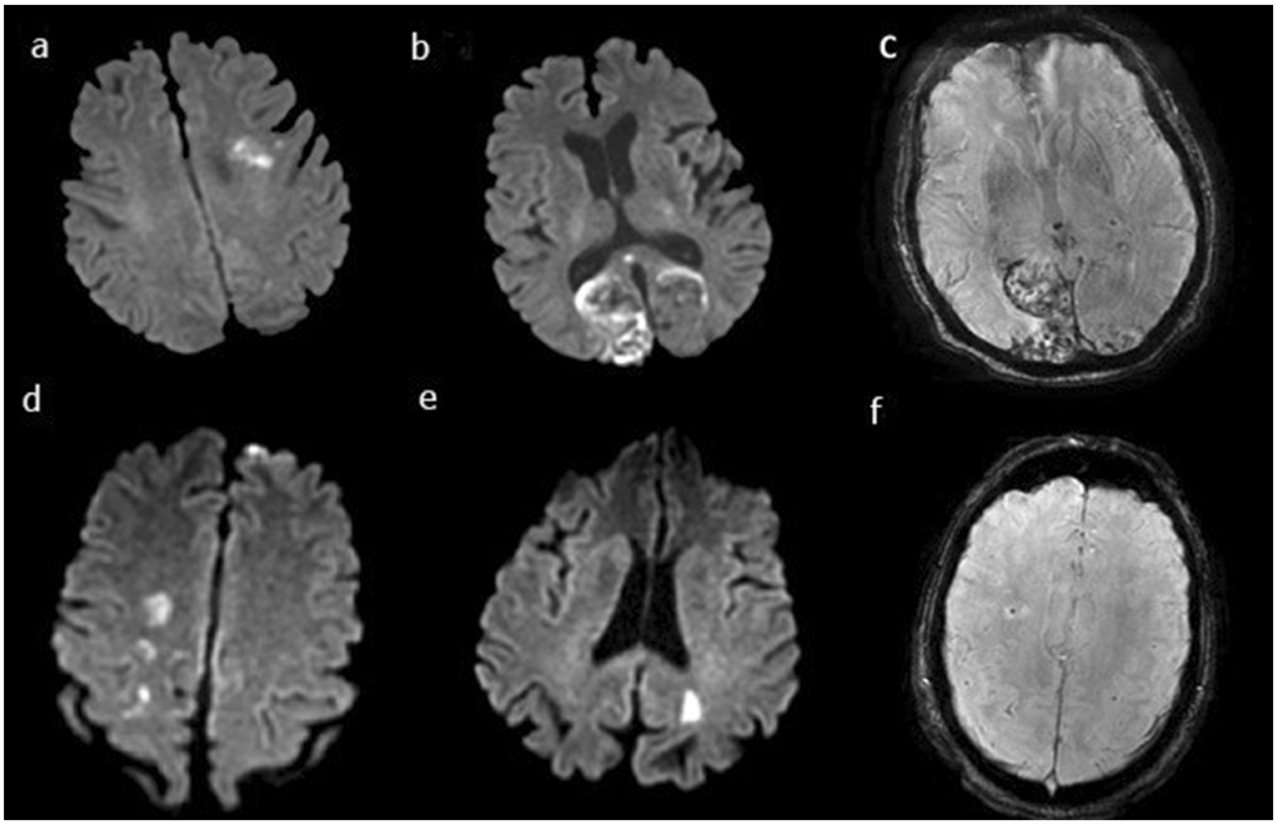


When waking up in the ICU, he was conscious without focal neurological deficits but with remarkable global altered mental status, fluctuating inattention, disorientation, inconsistent speech, and psychomotor agitation that required risperidone.

Systemic, infectious, and toxic-metabolic conditions were excluded. An electroencephalogram showed moderate encephalopathy and a brain MRI revealed acute bilateral ischemic lesions affecting both frontal and left occipital lobes, with several microhemorrhagic foci (Fig. 1d-f). Cardioembolic source and acquired antiphospholipid syndrome were ruled out. COVID-19-associated coagulopathy was suspected as the possible main etiology of the strokes, supported by the pulmonary embolism as another thrombotic complication.

\section{Discussion}

A high frequency of cerebrovascular events is being reported in COVID-19 patients, with a prevalence of around 5\% [7]. Owing to the recency of the pandemic, the available evidence of this association is based on small series and must be regarded cautiously. The strokes are frequently labeled as cryptogenic, but diagnostic investigations could not be completed in some COVID-19 patients and that may contribute to this high rate. Different mechanisms of stroke appear to be directly related to COVID-19 and are being progressively reported [4]. This includes a hypercoagulable state possibly linked to the viral invasion of the vascular endothelium, which may trigger activation of the complement system leading to thrombotic and inflammatory cascades $[3,4]$. Up to $20-55 \%$ of COVID-19 hospitalized patients may present this coagulopathy [3], but those with concomitant stroke show more significant disturbances (mostly higher D-dimer levels), suggesting that this state may underlie much of stroke in this disease [5]. Furthermore, some critically ill COVID-19 patients exhibit a coagulopathy consistent with disseminated intravascular coagulation [5], as in case 1.

To our knowledge, acute confusional state and encephalopathy have been described in COVID-19 patients related to the systemic condition, without a primary neurological cause $[2$, 8]. Although the most common findings in stroke are focal neurological deficits, ischemic lesions in those patients might be due to multifocal and distal vessel occlusions which could more frequently manifest as an altered mental status [9]. Additionally, this could be especially relevant in younger patients without previous comorbidities, less prone to present altered mental status during an acute disease, as in the cases we report.

In view of the above and supported by our evidence, we consider important to rule out cerebrovascular entities by performing periodic neurological examinations preceded by transient withdrawal of sedatives in those intubated COVID-
19 patients with suspicion of COVID-19-associated coagulopathy. We suggest performing a brain MRI in patients with acutely altered mental status on examination and in those who cannot be clinically evaluated [8].

Full-dose anticoagulation would probably be indicated and could prevent complications in these patients, being necessary more studies to confirm this supposition [10].

\section{Conclusions}

In conclusion, acutely altered mental status might be the main manifestation of multiple brain infarctions in critically ill COVID-19 patients and should be specially considered in those with suspected COVID-19-associated coagulopathy. Full-dose anticoagulation and clinical-radiological monitoring might reduce neurological consequences in them.

Author contribution Carolina Díaz-Pérez, MD, designed and conceptualized the study, analyzed the data, and drafted the manuscript for intellectual content. Carmen Ramos, MD, designed and conceptualized the study, analyzed the data, and drafted the manuscript for intellectual content. Alberto LópezCruz, MD, had a major role in the acquisition of data. José Muñoz Olmedo, MD, had major role in the acquisition of data. Jimena Lázaro González, MD, had a major role in the acquisition of data. Enrique de Vega-Ríos, MD, had a major role in the acquisition of data. Carmen González-Ávila, MD, had a major role in the acquisition of data. Carlos Hervás, MD, had a major role in the acquisition of data. Santiago Trillo, MD, $\mathrm{PhD}$, interpreted the data and revised the manuscript for intellectual content. José Vivancos, $\mathrm{MD}, \mathrm{PhD}$, interpreted the data and revised the manuscript for intellectual content.

\section{Compliance with ethical standards}

Due to the fact that it is a case report, it was not presented to the institutional Review Board or other ethics committee. Verbal informed consent was obtained from the patient's relatives to the submission of the case report.

Conflict of interest The authors declare that they have no conflict of interest.

\section{References}

1. Harapan H, Itoh N, Yufika A et al (2020) Coronavirus disease 2019 (COVID-19): a literature review. J Infect Public Health 13(5):667673. https://doi.org/10.1016/j.jiph.2020.03.019

2. Mao L, Jin H, Wang M et al (2020) Neurologic manifestations of hospitalized patients with coronavirus disease 2019 in Wuhan, China. JAMA Neurol 77(6):1-9. https://doi.org/10.1001/ jamaneurol.2020.1127

3. Spiezia L, Boscolo A, Poletto F et al (2020) COVID-19-related severe hypercoagulability in patients admitted to intensive care unit 
for acute respiratory failure. Thromb Haemost 120(6):998-1000. https://doi.org/10.1055/s-0040-1710018

4. Spence JD et al (2020) Mechanisms of stroke in COVID-19. Cerebrovasc Dis 20:1-8. https://doi.org/10.1159/000509581

5. Divani AA et al (2020) Coronavirus disease 2019 and stroke: clinical manifestations and pathophysiological insights. J Stroke Cerebrovasc Dis 29(8):104941. https://doi.org/10.1016/j. jstrokecerebrovasdis.2020.104941

6. Levi M, Toh CH, Thachil J et al (2009) Guidelines for the diagnosis and management of disseminated intravascular coagulation. British Committee for Standards in Haematology. Br J Haematol 145:24 33. https://doi.org/10.1111/j.1365-2141.2009.07600.x

7. Qureshi AI, Abd-Allah F, Al-Senani F, Aytac E, Borhani-Haghighi A, Ciccone A et al (2020) Management of acute ischemic stroke in patients with COVID-19 infection: report of an international panel.
Int J Stroke 15(5):540-554. https://doi.org/10.1177/ 1747493020923234

8. Helms J, Kremer S, Merdji H et al (2020) Neurologic features in severe SARS-CoV-2 infection. N Engl J Med 382(23):2268-2270. https://doi.org/10.1056/nejmc2008597

9. Ferro JM, Caeiro L, Verdelho A (2002) Delirium in acute stroke. Curr Opin Neurol 15(1):51-55. https://doi.org/10.1097/00019052200202000-00009

10. Tang N, Bai H, Chen X, Gong J, Li D, Sun Z (2020) Anticoagulant treatment is associated with decreased mortality in severe coronavirus disease 2019 patients with coagulopathy. J Thromb Haemost 18(5):1094-1099. https://doi.org/10.1111/jth.14817

Publisher's note Springer Nature remains neutral with regard to jurisdictional claims in published maps and institutional affiliations. 